\title{
3D Visibility Representations of Complete Graphs
}

\author{
Jan Štola \\ Department of Applied Mathematics, Charles University \\ Malostranské nám. 25, Prague, Czech Republic \\ Jan.Stola@mff. cuni.cz
}

\begin{abstract}
This paper continues the study of $3 \mathrm{D}$ visibility representations of complete graphs where vertices are represented by equal convex polygons lying in planes parallel to the $x y$-plane. Edges correspond to the $z$-parallel visibility among these polygons.

We give several bounds on the size of the largest complete graph that has a $3 \mathrm{D}$ visibility representation with particular properties. Namely we improve the best known lower bound for representations by regular $n$ gons from $\left\lfloor\frac{n+1}{2}\right\rfloor+2$ to $n+1$ and the upper bound from $2^{2^{n}}$ to $\left(\begin{array}{l}6 n-3 \\ 3 n-1\end{array}\right)-3$.
\end{abstract}

\section{Introduction}

In this paper we study visibility representations of graphs. Two-dimensional variants of these representations were intesively studied in the past because of their connection to the VLSI routing and circuit board layout 8, [10. Particularly the rectangle visibility graphs (axis-aligned rectangles in the plane with axis-aligned visibility segments among them) received wide attention (see for example [5] and [7) in the last decade.

It is natural to generalize these representations into three dimensions. Twodimensional Euclidean sets act as vertices there. The sets are situated in the planes parallel to the $x y$-plane. Two vertices are connected if and only if they can see each other in the direction that is orthogonal to their planes.

Fekete et al. study representations by squares and rectangles. In [6] and [9] it is shown that $K_{7}$ can be represented by equal squares but $K_{8}$ cannot. Similarly $K_{22}$ has a representation by rectangles of different shapes but $K_{56}$ has not. Alt, Godeau and Whitesides [1] prove that there doesn't exist a convex polygon $P$ such that any complete graph has a visibility representation by shifted copies of $P$. Particularly $K_{m}$ for $m>2^{2^{n}}$ cannot be represented by convex $n$-gons. They also describe a representation of any graph on $n$ vertices by (possibly different) polygons with at most $2 n$ sides. Babilon et al. [2] are interested in the maximum size of a clique represented by regular $n$-gons and present a lower bound $\left\lfloor\frac{n+1}{2}\right\rfloor+2$.

Cobos et al. [4] extend visibility representations even into the higher dimensions and characterize the dimensionality (e.g. the lowest dimension in which a representation of the given graph exists) of several classes of graphs. 
We continue in the study of the maximum size of a complete graph with a visibility representation where vertices are represented by shifted copies of a given (open) polygon. It can be easily shown (see for example 1]) that a complete graph of an arbitrary size can be represented by any non-convex polygon. So, our attention is restricted to convex polygons only. Therefore the term polygon means convex polygon in the sequel.

Let $s(P)$ denote the maximum size of a complete graph with a visibility representation by copies of the polygon $P$. Denote by $R_{n}$ a regular $n$-gon. It was shown in [1] and [2] that $\left\lfloor\frac{n+1}{2}\right\rfloor+2 \leq s\left(R_{n}\right) \leq 2^{2^{n}}$. We improve both sides of this estimate.

\section{Coordinates}

Let's remind the notion of polygon coordinates in a visibility representation (introduced in [2]). Firstly note that because of the openness of the polygons used in the representation we can expect that no two projections (into the plane $z=0$ ) of sides of the polygons lie in the same line. Let $P$ be a projection of some polygon into the plane $z=0$. Denote by $\boldsymbol{u}_{i}, i \in\{1, \ldots, n\}$ the unitary vector orthogonal to the $i$-th side of $P$. We choose the orientation of $\boldsymbol{u}_{i}$ such that $\boldsymbol{u}_{i}$ aims out of $P$.

Note that the definition of $\boldsymbol{u}_{i}$ doesn't depend on the selection of $P$ because all polygons in the representation are shifted copies of the same polygon.

Now consider projections of $i$-th sides of all polygons from the representation. The $i$-th coordinate of the polygon $P$ is the integer number that denotes the order (in the direction of $\boldsymbol{u}_{i}$ ) of the projection of the $i$-th side of $P$ among the projections of $i$-th sides of the other polygons.

The polygon coordinates allow us to recognize configurations that cannot appear in a representation of a complete graph.

Lemma 1. 2] Let $P, Q, R$ be $n$-gons whose $i$-th coordinates are monotone for every $i \in\{1, \ldots, n\}$. If the $z$-coordinates of those polygons are also monotone then $P$ cannot see $R$ along the $z$-axis.

\section{Relationships}

It is important to understand that individual coordinates of polygons are not independent.

Lemma 2. Let $P$ and $Q$ be polygons from a visibility representation. Let $\left(p_{1}, \ldots\right.$, $\left.p_{n}\right)$ and $\left(q_{1}, \ldots, q_{n}\right)$ be their coordinates. There exist $i, j \in\{1, \ldots, n\}, i<j$ such that one of the following conditions is satisfied:

$-p_{k}<q_{k}$ for $k \in\{i, \ldots, j-1\}$ and $p_{k}>q_{k}$ otherwise

- $p_{k}>q_{k}$ for $k \in\{i, \ldots, j-1\}$ and $p_{k}<q_{k}$ otherwise 
Proof. Let $Q^{\prime}$ be a projection of $Q$ into the plane of $P$. Denote by $\boldsymbol{u}$ the translation vector from $P$ to $Q^{\prime}$ and by $l_{k}$ the line that goes through the origin and is parallel to the $k$-th side of $P$. Let $\boldsymbol{u}_{k}$ be the vector from the definition of polygon coordinates. The line $l_{k}$ (that is orthogonal to $\boldsymbol{u}_{k}$ ) divides the plane into two half planes. We call them positive and negative depending on the fact whether $\boldsymbol{u}_{k}$ points into them or not. It is clear that $p_{k}<q_{k}$ if and only if $\boldsymbol{u}$ points into the positive half plane.

Now consider all lines $l_{k}$ together. The polygon $P$ is convex. Therefore one of the following two possibilities must occur.

- There exist $i$ and $j$ such that $\boldsymbol{u}$ lies in the positive half planes that correspond to lines $l_{k}, i \leq k<j$ and in the negative half planes that correspond to the other lines.

- There exist $i$ and $j$ such that $\boldsymbol{u}$ lies in the negative half planes that correspond to lines $l_{k}, i \leq k<j$ and in the positive half planes that correspond to the other lines.

And that is a reformulation of what we want to prove.

Definition 1. Let $P$ and $Q$ be polygons in a visibility representation. Denote by $i$ and $j$ the numbers from the previous lemma. We say that $P$ has a relationship $(i, j)$ to $Q$ if the first condition is satisfied. Otherwise we say that $P$ has to $Q$ a relationship $(j, i)$. Finally the relationship between $P$ and $Q$ is the relationship of the polygon with the smaller z-coordinate to the other one.

Consider relationships in a representation by the given polygon $P$. Each relationship corresponds to one part of the plane divided by cuts (going through the origin) that are parallel to the individual sides of $P$. For example we have $2 n$ relationships for a general $n$-gon or for a regular $2 n$-gon. We denote this number by $r(P)$.

Now we can reformulate Lemma 1 . in terms of relationships.

Lemma 3. Let $P, Q, R$ be polygons from a visibility representation. If $P$ has to $Q$ the same relationship as $Q$ has to $R$ then if $Q$ lies (according to the zcoordinate) between $P$ and $R$ then $P$ cannot see $R$.

\section{Upper Bounds}

In this section we present several upper bounds on the size of a complete graph with a visibility representation with particular properties. Firstly we improve the upper bound for $s\left(R_{n}\right)$ by using the notion of relationships.

Theorem 1. If $P$ is a polygon then $s(P) \leq\lfloor$ e.r $(P) !\rfloor$.

Proof. We proceed by induction on $r(P)$. Let's suppose that there are $k+1$ relationships in the given visibility representation. Divide polygons into sets 
$M_{1}, \ldots, M_{k+1}$ according to their relationship to the polygon with the maximum $z$-coordinate.

If $r$ is a relationship that corresponds to the set $M_{i}$ then there are no two polygons in $M_{i}$ with this relationship. Otherwise these two polygons together with the one with the maximum $z$-coordinate would fulfill presumptions of Lemma 3. Hence, there are at most $k$ types of relationships among polygons in $M_{i}$. Therefore $\left|M_{i}\right| \leq\lfloor e . k !\rfloor$ and there is at most $1+(k+1)\lfloor e . k !\rfloor=\lfloor e .(k+1) !\rfloor$ polygons in the whole representation.

It remains to prove the simplest case $k=1$. If there is only one type of relationship in the representation then there can be at most $2=\lfloor e .1 !\rfloor$ polygons. Otherwise we would be able to apply Lemma 3 . to any triple of polygons.

Later in the paper we derive an estimate with a better asymptotic behavior than the previous one but the previous estimate of $s\left(R_{n}\right)$ gives the best known upper bounds for $n$ equal to $5,6,8$ and 10 .

For the sake of simplicity we work in the rest of this paper with objects in different parallel planes as if they are in the same plane. From the formal point of view such a description should be understood as an operation on projections into a common plane and projections of a result back to the individual planes.

In the rest of this section we denote by $X_{i}$ the first vertex of the $i$-th polygon from the representation. We also say that polygons $P_{1}, \ldots, P_{k}$ are in $z$-order if they are ordered according to their increasing $z$-coordinate.

Lemma 4. Let polygons $P_{1}, \ldots, P_{k}$ (in $z$-order) form a visibility representation of $K_{k}$. If $X_{1} \ldots X_{k} X_{1}$ form a boundary of a convex polygon then $k \leq r\left(P_{1}\right)+2$.

Proof. Denote by $\boldsymbol{u}_{i}$ the vector $X_{i+1}-X_{i} . X_{1} X_{2} \ldots X_{k} X_{1}$ is a boundary of a convex polygon. Therefore $\left(\boldsymbol{u}_{i}\right)_{i=1}^{k-1}$ is a sequence of vectors that rotate around the origin by less than $2 \pi$.

The vector $\boldsymbol{u}_{i}$ is clearly a translation vector from $P_{i}$ to $P_{i+1}$. This vector determines the relationship between $P_{i}$ and $P_{i+1}$. Because of the rotation around the origin and Lemma 3 . there cannot be two equal relationships between polygons $P_{i}$ and $P_{i+1}$, with one exception - the relationship between $P_{1}$ and $P_{2}$ could be the same as the relationship between $P_{k-1}$ and $P_{k}$. Hence $k \leq r\left(P_{1}\right)+2$.

Regular $(2 n+1)$-gons can have two types of relationships $(i, j):(j-i) \bmod$ $(2 n+1)$ could be either $n$ or $n+1$. For $(4 n+1)$-gons the first situation corresponds to the pull of the upper polygon by a vertex and the second to the pull by an edge. The opposite holds for $(4 n+3)$-gons. That is why we call such relationships edge-relationships and vertex-relationships.

Definition 2. Let $V$ be a vertex of a regular $(2 n+1)$-gon $P$ with the center $S_{P}$. Denote by $P^{\prime}$ the copy of $P$ shifted by the vector $S_{P} V$. We call the relationship of $P$ to $P^{\prime} V$-relationship (or the relationship $V$ ).

Let $E$ be an edge of $P$. If we replace $V$ in the previous paragraph by the center of $E$ we get the definition of E-relationship (the relationship $E$ ). The following two lemmas give us the basic properties of these relationships. 


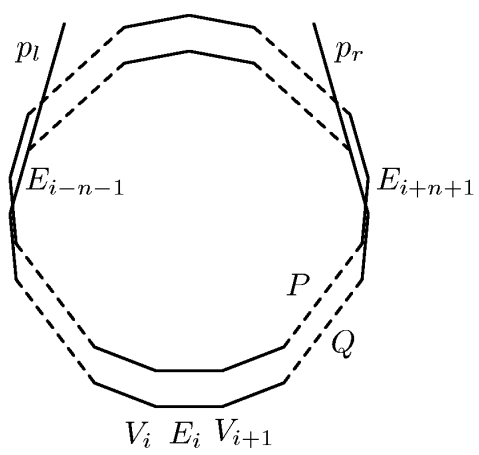

Lemma 5. Let $P, Q, R$ (in z-order) be regular $(4 n+1)$-gons from a visibility representation. Denote by $V_{i}\left(E_{i}\right)$ the $i$-th vertex (edge) of $P$. If $P$ and $Q$ have $E_{i}$-relationship and $Q$ and $R$ have $V_{i+1}$-relationship then $P$ and $R$ cannot see each other.

Proof. Let's rotate the whole situation such that the vector $V_{i} V_{i+1}$ is parallel to the vector $(1,0)$. Denote by $p_{l}\left(p_{r}\right)$ the line that contains $E_{i-n-1}\left(E_{i+n+1}\right)$ - the edge going from the vertex with the smallest (biggest) $x$-coordinate that goes away from $E_{i}$. It is clear that $P$ and $R$ cannot see each other if the translation vector between $Q$ and $R$ is lying between $p_{r}$ and $p_{l}$, but the translation vector that corresponds to $V_{i+1}$-relationship has to lie between $V_{i+n+2} V_{i+n+1}$ and $V_{i-n} V_{i-n+1}$.
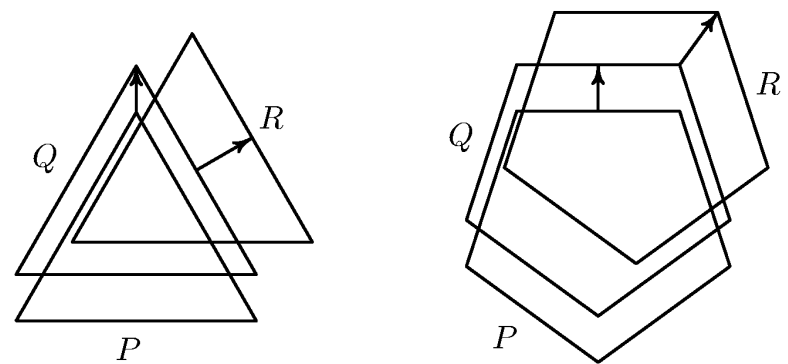

Lemma 6. Let $P, Q, R$ (in z-order) be regular $(4 n+3)$-gons from a visibility representation. Denote by $V_{i}\left(E_{i}\right)$ the $i$-th vertex (edge) of $P$. If $P$ and $Q$ have $V_{i}$-relationship and $Q$ and $R$ have $E_{i}$-relationship then $P$ and $R$ cannot see each other.

Proof. We proceed in the same way as in the previous proof. 
Lemma 4. gives an upper bound $2 n+2$ for regular $2 n$-gons. Using Lemmas 5 . and 6 . we can prove the same bound also for regular $(2 n+1)$-gons.

Lemma 7. Let regular $(2 n+1)$-gons $P_{1}, \ldots, P_{k}$ (in z-order) form a visibility representation of $K_{k}$. If the centers of the polygons form a boundary of a convex polygon then $k \leq 2 n+2$.

Proof. We prove the lemma for $(4 n+1)$-gons. The proof for $(4 n+3)$-gons is similar.

Using the same argument as in the proof of Lemma 4. we can show that there cannot be the same relationships between polygons $P_{i}$ and $P_{i+1}$. Again with one exception - the relationship between $P_{1}$ and $P_{2}$ could be the same as the relationship between $P_{k-1}$ and $P_{k}$.

Without loss of generality we can assume that the vertices and edges are numbered such that the relationship between $P_{1}$ and $P_{2}$ is $V_{1}$ or $E_{1}$ and that the sequence of relationships between $P_{i}$ and $P_{i+1}$ is a subsequence of $V_{1} E_{1} V_{2} \ldots$ $E_{m} V_{1}$ or $E_{1} V_{2} E_{2} \ldots E_{m} V_{1} E_{1}$, where $m=4 n+1$. Due to Lemma 5 . we know that this sequence doesn't contain any pair $E_{i} V_{i+1}$.

Hence, it is sufficient to show that a subsequence avoiding mentioned pair that is chosen

- from $E_{1} V_{2} \ldots E_{m} V_{1}$ has length at most $m$,

- from $V_{1} E_{1} V_{2} \ldots E_{m} V_{1}$ has length at most $m+1$.

We proceed by induction, for $m=1$ the claim holds because we have to leave at least one relationship from the sequences $E_{1} V_{2}$ and $V_{1} E_{1} V_{2}$.

Let's consider what can remain from the first two relationships in the first case. If we omit $E_{1}$ then by the second case the length of the subsequence is at most $m$. If we omit $V_{2}$ we get the first case for a shorter sequence and again the length at most $m$.

In the second case we have to consider the first three relationships. If we omit $V_{1}$ then by the first case the length of the subsequence is at most $m$. If we omit $E_{1}$ then we get the second case for a shorter sequence and the length at most $m+1$. Finally if we omit $V_{2}$ we get again the length at most $m+1$ by the first case.

We demanded that corresponding points of polygons lie in a convex position and that polygons are ordered according to their $z$-coordinates. Let's see how the situation changes when we remove the second requirement.

Lemma 8. Let polygons $P_{1}, \ldots, P_{k}$ form a visibility representation of $K_{k}$. If $X_{1} \ldots X_{k} X_{1}$ form a boundary of a convex polygon then $k \leq 3 r\left(P_{1}\right)$.

Proof. Let $\boldsymbol{u}_{i}$ has the same meaning as in the proof of Lemma 4 . Denote by $r_{i}$ the relationship that corresponds to the translation vector $\boldsymbol{u}_{i} \cdot\left(\boldsymbol{u}_{i}\right)_{i=1}^{k}$ is a sequence of vectors that rotate around the origin by less than $2 \pi$. Therefore the relationships 
that occur multiple times in the sequence $\left(r_{i}\right)$ must have all occurrences adjacent (after possible renumbering) 1

If the sequence is longer than $3 r\left(P_{1}\right)$ then in the sequence $\left(r_{i}\right)$ there must exist a constant segment of length at least 4 . This segment corresponds to the sequence of at least 5 polygons with the same relationships. It is always possible to choose from those polygons a subsequence of length 3 with monotone $z$ coordinates. According to Lemma 3. polygons with this property cannot lie in a representation of a complete graph. Therefore $k \leq 3 r\left(P_{1}\right)$.

Again we can use Lemmas 5. and 6. to improve the estimate for $(2 n+1)$-gons.

Lemma 9. Let regular $(2 n+1)$-gons $P_{1}, \ldots, P_{k}$ form a visibility representation of $K_{k}$. If the centers of the polygons form a boundary of a convex polygon then $k \leq 3(2 n+1)$.

Proof. We proceed in the same way as in the previous proof to the definition of the sequence $\left(r_{i}\right)_{i=1}^{k}$. There we have to utilize the fact that some relationships cannot be adjacent. We show the proof only for $(4 n+1)$-gons because the argument for $(4 n+3)$-gons is similar.

There cannot be the relationship $E_{i}$ immediately followed by the relationship $V_{i+1}$ or $V_{i}$ in the sequence $\left(r_{i}\right)$. Without loss of generality we can assume that vectors $\boldsymbol{u}_{i}$ turn from $E_{i}$ to $V_{i+1}$, otherwise we can take the opposite numbering of polygons.

Let's group the relationships into $4 n+1$ groups such that there are relationships $E_{i}$ and $V_{i+1}$ in the $i$-th group. Members from the same group are (after possible renumbering) adjacent in the sequence $\left(r_{i}\right)$. If the sequence is longer than $3(4 n+1)$ then there must be a segment of length at least 4 whose members belong into the $i$-th group for some $i \in\{1, \ldots, 4 n+1\}$. By the construction the relationships $E_{i}$ are before $V_{i+1}$ in this segment. The segment corresponds to the sequence of at least 5 polygons from which we can chose a subsequence of 3 polygons with monotone $z$-coordinates. These three polygons have one type of relationship or $E_{i}$-relationship followed by $V_{i+1}$-relationship. According to Lemma 3. or 5. such polygons cannot lie in a representation of a complete graph.

If we remove the requirement for a convex position of corresponding points of polygons we get a general estimate of $s(P)$.

Theorem 2. $s(P) \leq\left(\begin{array}{c}6 r(P)-3 \\ 3 r(P)-1\end{array}\right)-3$.

Proof. Due to the upper bound for the Erdös-Szekeres problem presented in [3] there exist among any $\left(\begin{array}{c}6 r(P)-3 \\ 3 r(P)-1\end{array}\right)-2$ points in the plane at least $3 r(P)+1$ points in a convex position.

Choose an arbitrary point of $P$. If we have a representation with more than $\left(\begin{array}{l}6 r(P)-3 \\ 3 r(P)-1\end{array}\right)-3$ polygons then there exists a group of at least $3 r(P)+1$ of them

${ }^{1}$ It could happen that a part of the occurrences lies at the start of the sequence and a part at the end. 
such that their points that correspond to the chosen point are in a convex position. According to Lemma 8. such a group cannot appear in a representation of a complete graph.

Particularly we improved the best known upper bound for $s\left(R_{n}\right)$.

Corollary 1. $s\left(R_{n}\right) \leq\left(\begin{array}{c}6 n-3 \\ 3 n-1\end{array}\right)-3 \approx 2^{6 n}$.

Proof. For even $n$ this result is a consequence of the previous theorem. If $n$ is odd then we can repeat the previous proof and use Lemma 9. instead of Lemma 8. at the end.

The last exponential estimate doesn't look very tight after the linear estimates from the rest of the paper. Below we show that its exponential behavior could not be improved by the used technique. So far, the only forbidden configuration was a triple of polygons where the middle one blocked the visibility between the other two polygons. There exist representations of an exponential size that don't contain this configuration.

Lemma 10. Let $P, Q, R$ (in z-order) be regular $n$-gons from a visibility representation. If the relationship between $P$ and $Q$ is different from the relationship between $Q$ and $R$ and

- for $n=4 k+1$ the relationship between $P$ and $Q$ is a vertex-relationship, or it is an E-relationship but the relationship between $Q$ and $R$ is not a $V$ relationship such that $V \in E$,

- for $n=4 k+3$ the relationship between $P$ and $Q$ is an edge-relationship, or it is a $V$-relationship but the relationship between $Q$ and $R$ is not an $E$ relationship such that $V \in E$,

then polygon $P$ can see $R$ provided that the translation vectors among $P, Q$ and $R$ are sufficiently small.

Proof. Let $(i, j)$ be the relationship between $P$ and $Q$. If the translation vector from $P$ to $Q$ is sufficiently small then the $i$-th side of $P$ intersects the $(i-1)$-th side of $Q$ and the $j$-th side of $Q$ intersects the $(j-1)$-th side of $P$. Denote by $p_{k}$ the line that contains the $k$-th side of $Q$. If the translation vector from $Q$ to $R$ lies in the opposite half plane given by the line $p_{i-1}$ (respectively $p_{j}$ ) than polygon $Q$ then $P$ and $R$ can see each other in the neighborhood of the $(i-1)$-th (resp. the $j$-th) side of $Q$.

Before we continue we must understand how to find for the given translation vector $\boldsymbol{u}$ all vectors that correspond to the same relationship. It is clear from the proof of Lemma 2. that those vectors are the ones between the sides that form the smallest positive and the smallest negative angle with $\boldsymbol{u}$.

With this information we can rewrite the first paragraph into the words of relationships. The situation is simple for $2 n$-gons because for their relationships $(i, j)$ we have $(j-i) \equiv n \bmod 2 n$. Hence, the $(i-1)$-th and $j$-th side form the 
smallest angle and translation vectors between them correspond to one relationship - the relationship between $P$ and $Q$. If $Q$ and $R$ have a different relationship then $P$ can see $R$ provided that the translation vectors are small.

We can use a similar argument for $(4 n+1)$-gons and a vertex-relationship or for $(4 n+3)$-gons and an edge-relationship between $P$ and $Q$.

The remaining situations are slightly more complicated because there are two sides between the $(i-1)$-th and the $j$-th side of $Q$. The translation vectors between those sides correspond to the relationships $V_{l} E_{l} V_{l+1}$ (for $(4 n+1)$-gons) or $E_{l-1} V_{l} E_{l}$ (for $(4 n+3)$-gons). Therefore $P$ can see $R$ if $Q$ and $R$ don't have a relationship adjacent to the relationship between $P$ and $Q$.

Theorem 3. There exists a visibility representation with $4^{n}$ (resp. $\left.4^{n+1}\right)$ regular $2 n$-gons (resp. $(2 n+1)$-gons) where every triple of polygons alone form a representation of $K_{3}$.

Proof. Let's choose $n$ adjacent relationships for $2 n$-gons or relationships $V_{1}, \ldots$, $V_{n+1}$ for $(2 n+1)$-gons. It is important that all corresponding translation vectors lie in one half plane.

We proceed by induction on the number of chosen relationships. If we have one relationship we place 4 polygons in a direction given by the chosen relationship and set their $z$-coordinates equal to $2,1,4$ and 3 subsequently.

Let's suppose that we have a construction for $k$ relationships. The $(k+1)$-th relationship corresponds to the translation vectors between two half lines $p_{l}$ and $p_{r}$. Consider projections (to the plane $z=0$ ) of the centers of all polygons from the construction. Draw two tangents to the convex hull of the projections - one parallel to $p_{l}$ and one parallel to $p_{r}$. Now we place a copy of the construction for $k$ relationships to the opposite quadrant determined by the tangents. We repeat the process three times.

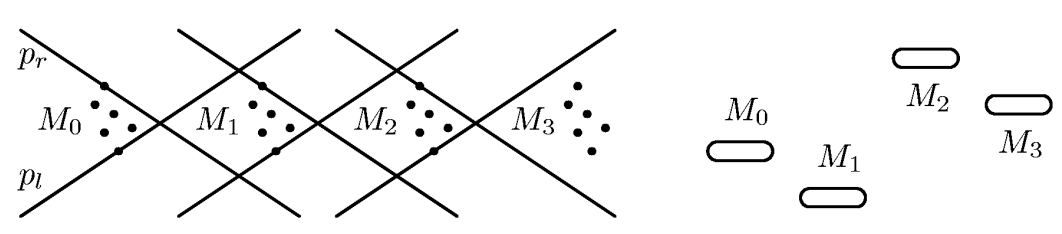

We have four copies of $4^{k}$ polygons. It is important that polygons from the different copies have $(k+1)$-th relationship. Now we place all polygons from the first copy below and from the third copy above the original polygons. The second copy is placed above all other polygons. Finally we proportionally shorten the distances among the centers of the polygons.

Let $P, Q, R$ (in $z$-order) be polygons from the construction. If $P$ and $Q$ don't have the same relationship as $Q$ and $R$ then they can see each other because of the previous lemma and the set of relationships considered. The mentioned 
relationships cannot be the same because we place individual copies in all steps such that there are no three polygons (in $z$-order) with $(k+1)$-th relationships among them.

\section{Lower Bound}

We improved the best known upper bound for $s\left(R_{n}\right)$ in the previous section. Now we concentrate on the lower bound.

Theorem 4. $s\left(R_{n}\right) \geq n+1$.

Proof. By [2] we have $s\left(R_{3}\right) \geq 14$. So, we can assume $n \geq 4$.

At first we describe a representation of $K_{n}$. Later we add the $(n+1)$-th polygon to obtain a representation of $K_{n+1}$.

Let $P$ be a regular $n$-gon whose vertices $v_{i}$ lie on the unitary circle centered at the origin e.g. $v_{i}=\left(\cos \frac{2 \pi}{n} i, \sin \frac{2 \pi}{n} i\right)$. Polygons $P_{i}$ are created by $1 / 2 \mathrm{long}$ shifts of $P$ in the direction of $\boldsymbol{u}_{i}=\left(\cos \left(\frac{2 \pi}{n} i+\alpha\right), \sin \left(\frac{2 \pi}{n} i+\alpha\right)\right)$, where $\alpha$ is an angle whose value is determined at the end of the proof. We claim that $P_{i}$ moved into planes $z=i$ form the desired representation of $K_{n}$.

It can be easily verified that we can obtain $P_{i+1}$ by a rotation of $P_{i}$ around the origin by $\frac{2 \pi}{n}$. Hence, $P_{0}$ can see $P_{k}$ if and only if $P_{l}$ can see $P_{k+l}, k \in$ $\{1, \ldots, n-1\}, l \in\{0, \ldots, n-1-k\}$. Therefore it is sufficient to show that $P_{0}$ can see all $P_{k}, k \in\{1, \ldots, n-1\}$.

Denote by $S_{i}$ the center of the polygon $P_{i}$. We claim that $P_{0}$ and $P_{k}$ can see each other through the intersection of their inscribed circles, particularly through the intersection $X$ that is in the opposite part (than points $S_{i}$ ) of the plane determined by the angle $S_{0} O S_{k}-O$ denotes the origin.

To prove that $P_{0}$ and $P_{k}$ can see each other through $X$ it is sufficient to show that $X$ is not inside circles circumscribed to the polygons $P_{j}, 0<j<k$, e.g. to show that it is at least 1 unit far from their centers.

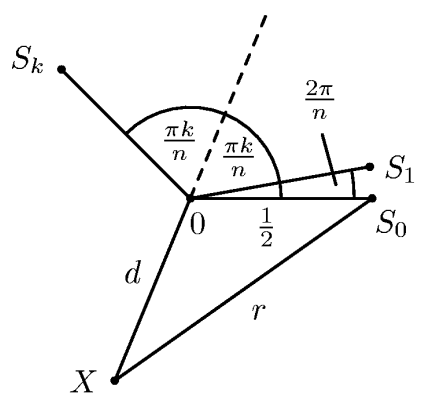

Let $r$ denote the radius of the circle inscribed to $P$. We have $r>1 / 2$ because $r=\cos \frac{\pi}{n}$ and $n \geq 4$. So, there exists an intersection of the inscribed circles 
outside the angle $S_{0} O S_{k}$ and the point $X$ is correctly defined. The nearest points from $S_{1}, \ldots, S_{k-1}$ are $S_{1}$ and $S_{k-1}$, both equally far. Therefore it remains to prove the inequality $\left|X S_{1}\right|>1$.

Let $d=|X O|$. According to the cosines laws we have

$$
\begin{aligned}
\left|X S_{1}\right|^{2} & =d^{2}+\frac{1}{4}-d \cos \left(\frac{n-k+2}{n} \pi\right) \\
r^{2} & =d^{2}+\frac{1}{4}-d \cos \left(\frac{n-k}{n} \pi\right)
\end{aligned}
$$

Hence, it is sufficient to show

$$
\begin{aligned}
1<\left|X S_{1}\right|^{2}= & r^{2}+d\left(\cos \left(\frac{n-k}{n} \pi\right)-\cos \left(\frac{n-k+2}{n} \pi\right)\right)= \\
& =\cos ^{2} \frac{\pi}{n}+2 d \sin \frac{\pi}{n} \sin \left(\frac{k-1}{n} \pi\right),
\end{aligned}
$$

for $k \in\{1, \ldots, n-1\}$. We can ignore the case $k=1$ because two adjacent polygons in this representation can see each other and we can write $\sin \frac{k-1}{n} \pi \geq$ $\sin \frac{\pi}{n}$ for the rest. Our inequality can be rewritten to

$$
1<\cos ^{2} \frac{\pi}{n}+2 d \sin ^{2} \frac{\pi}{n} \equiv 0<(2 d-1) \sin ^{2} \frac{\pi}{n} \equiv d>\frac{1}{2} .
$$

The estimate $\sin \frac{k-1}{n} \pi \geq \sin \frac{\pi}{n}$ is sharp for $k \neq 2$. Hence, for $k \neq 2$ it suffices to show $d \geq 1 / 2$. The inequality $d<1 / 2$ leads to the following contradiction:

$$
\cos ^{2} \frac{\pi}{n}=r^{2}=d^{2}+\frac{1}{4}+d \cos \frac{k \pi}{n}<\frac{1}{2}+\frac{1}{2} \cos \frac{2 \pi}{n}=\cos ^{2} \frac{\pi}{n} .
$$

We have proved that $d \geq 1 / 2$. It remains to solve the case $k=2$ and $d=1 / 2$. This happens when the circles inscribed into the polygons $P_{0}$ and $P_{2}$ intersect exactly on the circle circumscribed to the polygon $P_{1}$. If there is not a vertex of the polygon $P_{1}$ in the intersection then polygons $P_{0}$ and $P_{2}$ still can see each other. We achieve this by the mysterious angle $\alpha$ from the beginning of the proof.

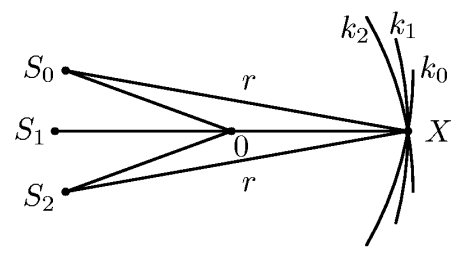

The described problematic situation could happen only when the vector $\boldsymbol{u}_{1}$ has the same direction as one of the vectors $-\boldsymbol{v}_{i}$. So, we have to choose the vector $\alpha$ to avoid a finite number of those critical values. 
Now we have a representation of $K_{n}$. We must add a new polygon to create a representation of $K_{n+1}$. We claim that such polygon could be a copy of $P_{0}$ shifted to the plane $z=n$. We could consider such a polygon to be a natural successor of $P_{n-1}$ in our construction - it can be created by a shift of $P$ by a vector $\boldsymbol{u}_{0}=\boldsymbol{u}_{n}=\left(\cos \left(\frac{2 \pi}{n} n+\alpha\right), \sin \left(\frac{2 \pi}{n} n+\alpha\right)\right)$. From this point of view it is clear that $P_{n}$ can see $P_{k}, k \in\{1, \ldots, n-1\}$ if and only if $P_{n-1}$ can see $P_{k-1}$ which is true. The only problem could be the visibility between $P_{0}$ and $P_{n}$.

For simplicity we rotate the whole situation such that the translation vector $\boldsymbol{u}_{n}$ is parallel to the vector $(1,0)$. The polygons $P_{0}$ and $P_{n}$ can see each other through the neighborhood of the vertex with the biggest $x$-coordinate. There cannot be a polygon that would break such a visibility because its translation vector $\boldsymbol{u}_{i}$ would have to have bigger $x$-coordinate than the vector $\boldsymbol{u}_{n}$ which is impossible because we rotated the situation such that this vector is parallel to $(1,0)$.

\section{Conclusion}

We derived several bounds for $s(P)$ for representations with particular properties and improved the lower bound for $s\left(R_{n}\right)$ from $\left\lfloor\frac{n+1}{2}\right\rfloor+2$ to $n+1$ and the upper bound from $2^{2^{n}}$ to $\left(\begin{array}{c}6 n-3 \\ 3 n-1\end{array}\right)-3 \approx 2^{6 n}$. In Theorem 3 . we showed that the exponential upper bound cannot be improved if we study only visibility in triples of polygons (e.g. if we look for one polygon that blocks the visibility between another two polygons).

\section{References}

1. H. Alt, M. Godau, S. Whitesides (1998): Universal 3-dimensional visibility representations for graphs, Comput. Geom. 9, 111-125

2. R. Babilon, H. Nyklová, O. Pangrác, J. Vondrák (1999): Visibility representations of complete graphs, Proc. Graph Drawing 99, 333-341

3. G. Tóth, P. Valtr (1998): Note on the Erdős-Szekeres theorem, Discrete Comput. Geom. 19(3), 457-459

4. F.J. Cobos, J.C. Dana, F. Hurtado, A. Márquez, F. Mateos (1995): On a visibility representation of graphs, Proc. Graph Drawing 95, 152-161

5. P. Bose, A. Dean, J. Hutchinson, T. Shermer (1996): On rectangle visibility graphs, Proc. Graph Drawing 96, 25-44

6. S.P. Fekete, M.E. Houle, S. Whitesides (1995): New results on a visibility representation of graphs in 3D, Proc. Graph Drawing 95, 234-241

7. J.P. Hutchinson, T. Shermer, A. Vince (1999): On representations of some thickness-two graphs, Comput. Geom. 13(3), 161-171

8. R. Tamassia, I.G. Tollis (1986): A unified approach to visibility representations of planar graphs, Discrete and Computational Geometry, 1:321-341

9. P. Bose, H. Everett, S. Fekete, A. Lubiw, H. Meijer, K. Romanik. T. Shermer, S. Whitesides (1993): On a visibility representation for graphs in three dimensions, Proc. Graph Drawing 93, 38-39

10. A. Dean, J. Hutchinson (1997): Rectangle-visibility representations of bipartite graphs, Discrete Appl. Math. 75(1), 9-25 